\title{
Siliceous mesostructured cellular foams/ poly(3-hydroxybutyrate-co-3-hydroxyhexanoate) composite biomaterials for bone regeneration
}

This article was published in the following Dove Press journal:

International Journal of Nanomedicine

20 October 2014

Number of times this article has been viewed

\begin{abstract}
Shengbing Yangl,*
Shuogui $\mathrm{Xu}^{2, *}$

Panyu Zhou ${ }^{2, *}$

jing Wang ${ }^{3}$

Honglue Tan ${ }^{4}$

Yang Liu ${ }^{5}$

TingTing Tang ${ }^{4}$

ChangSheng Liu'1,3,5

'The State Key Laboratory of Bioreactor Engineering, East China University of Science and Technology, Shanghai, People's Republic of China; ${ }^{2}$ Changhai Hospital, Department of Orthopedics, the Second Military Medical University, Shanghai, People's Republic of China; ${ }^{3}$ Key Laboratory for Ultrafine Materials of Ministry of Education, East China University of Science and Technology, Shanghai, People's Republic of China; ${ }^{4}$ Shanghai Key Laboratory of Orthopedic Implant, Department of Orthopedic Surgery, Shanghai 9th People's Hospital, Shanghai Jiao Tong University School of Medicine China, Shanghai, People's Republic of China; ${ }^{5}$ Engineering Research Center for Biomedical Materials of Ministry of Education, East China University of Science and Technology, Shanghai, People's Republic of China
\end{abstract}

*These authors contributed equally to this work

Correspondence: ChangSheng Liu The State Key Laboratory for Bioreactor Engineering, East China University of Science and Technology, Shanghai 200237, People's Republic of China

Tel +862164251308

Fax +862164251358

Email csliu@sh163.net

\begin{abstract}
Osteoinductive and biodegradable composite biomaterials for bone regeneration were prepared by combining poly(3-hydroxybutyrate-co-3-hydroxyhexanoate) (PHBHHx) with siliceous mesostructured cellular foams (SMC), using the porogen leaching method. Surface hydrophilicity, morphology, and recombinant human bone morphogenetic protein 2 adsorption/ release behavior of the SMC/PHBHHx scaffolds were analyzed. Results of scanning electron microscopy indicated that the SMC was uniformly dispersed in the PHBHHx scaffolds, and SMC modification scaffolds have an interconnected porous architecture with pore sizes ranging from 200 to $400 \mu \mathrm{m}$. The measurements of the water contact angles suggested that the incorporation of SMC into PHBHHx improves the hydrophilicity of the composite. In vitro studies with simulated body fluid show great improvements to bioactivity and biodegradability versus pure PHBHHx scaffolds. Cell adhesion and cell proliferation on the scaffolds was also evaluated, and the new tools provide a better environment for human mesenchymal stem cell attachment, spreading, proliferation, and osteogenic differentiation on PHBHHx scaffolds. Moreover, micro-computed tomography and histological evaluation confirmed that the SMC/PHBHHx scaffolds improved the efficiency of new bone regeneration with excellent biocompatibility and biodegradability and faster and more effective osteogenesis in vivo.
\end{abstract}

Keywords: stem cells, mesoporous, scaffolds, bone regeneration, rhBMP-2

\section{Introduction}

One approach to bone regeneration is the use of three-dimensional (3D) scaffolds to provide a template for the formation of the bone extracellular matrix (ECM). ${ }^{1,2}$ Highly porous scaffolds promote cell adhesion and proliferation and have an interconnected pore structure that allows nutrients to penetrate into the scaffold in vitro and in vivo. ${ }^{3-5}$ Numerous polymers are widely used in many tissue engineering applications, including bone regeneration, for their good biocompatibility and their ability to retain their physical and mechanical properties long enough to allow tissue growth. ${ }^{6-9}$ However, hydrophobic polymers have bioactivity and osteoconductivity that are suboptimal for a substrate because of their relatively low hydrophilicity compared with natural ECM..$^{10,11}$ Thus, it is necessary to add bioactive materials to improve the biological performance of the scaffold.

Incorporating or coating therapeutic ingredients such as endogenous osteoinductive growth factors (eg, bone morphogenetic proteins [BMPs]) into porous scaffolds for enhancing bone defect repair has been reported. ${ }^{4}$ Ideally, this means engineering a multiphase material that closely mimics the ECM of natural bone and controls the release kinetics of growth factor after implantation. 
Recently, mesoporous materials ${ }^{12,13}$ with pores from 2 to $50 \mathrm{~nm}$ have been shown to stimulate the formation and deposition of calcium phosphates from a physiological solution ${ }^{14,15}$ to promote the differentiation and proliferation of osteoblasts, resulting in an enhanced bone matrix interface strength. ${ }^{16,17}$ However, mesoporous materials, similar to other inorganic ceramic materials, are highly brittle and inflexible and can hamper the regeneration process. ${ }^{16}$ To alleviate some of these inherent issues, composite materials comprising a mesoparticulate and a biodegradable polymeric phase were developed in the field of bone regeneration. These materials not only mimic the major ECM of bone but also combine the advantages and minimize the limitations intrinsic to each material choice. ${ }^{18}$ For example, Zhu et al, who studied poly(L-lactic acid), showed that the addition of mesoporous bioactive glasses (MBG) results in enhanced attachment, spreading, and proliferation of rabbit bone marrow stromal cells. ${ }^{19} \mathrm{Wu}$ et al fabricated a composite composed of poly(lactic-glycolic acid) and MBG. Surface hydrophilicity, apatite-formation ability, and human osteoblastic-like cell attachment, spreading and proliferation, and differentiation were all significantly increased by the addition of MBG. ${ }^{18}$

Thus, we speculated that the microstructure of the bioactive inorganic phase plays an important role in bioactivity. However, because the pore diameter of MBG is smaller than the BMP-2 molecular size, the initial burst release of bioactive ingredients from the composite scaffolds will reduce the bioutility of the loaded growth factors. Siliceous mesostructured cellular foams (SMC) possess a unique, highly ordered structure with pore sizes even larger than $20 \mathrm{~nm}$ that are similar or larger than most enzyme molecules. Therefore, the large internal surface area of these supports can be accessible to BMPs, and the presence of various active (eg, silanol) groups on the surface also can facilitate protein adsorption, resulting in high BMP loads, and prolong its release time. Therefore, the objective of this study was to fabricate an SMC/poly(3-hydroxybutyrateco-3-hydroxyhexanoate) (PHBHHx) composite that mimics the structure of the ECM, using the porogen leaching process. We characterized microscopic and macroscopic structures in vitro and their biocompatibility and osteogenesis in vivo.

\section{Materials and methods}

\section{Materials}

The 1,3,5-triethylbenzene, tetraethyl orthosilicate, ethyl alcohol, $\mathrm{HCl}$, triblock copolymer Pluronic $\mathrm{P} 123\left(\mathrm{EO}_{20}-\mathrm{PO}_{70}-\mathrm{EO}_{20}\right.$, $\mathrm{Mw}=5800), \mathrm{NaCl}$, and chloroform were purchased from Sigma-Aldrich (St Louis, MO, USA) and were used without further purification. The PHBHHx (containing $12 \mathrm{~mol} \%$
HHx), with a molecular weight of 440,000, was donated by Professor GQ Chen from the Department of Biological Sciences and Biotechnology, Tsinghua University, Beijing, People's Republic of China.

\section{Synthesis of SMC and fabrication of 3D scaffolds}

The SMC was synthesized according to a previously reported method. ${ }^{20}$ Typically, Pluronic P123 (BASF, Frankfurt, Germany; $2.0 \mathrm{~g}, 0.4 \mathrm{mmol}$ ) was dissolved in $1.6 \mathrm{M} \mathrm{HCl}$ $(75 \mathrm{~mL}, 120 \mathrm{mmol} \mathrm{HCl})$ at room temperature while being stirred in a beaker covered with a watch glass. 1,3,5triethylbenzene $(2.0 \mathrm{~g}, 17 \mathrm{mmol})$ was then added, and the mixture was heated to $40^{\circ} \mathrm{C}$. After 60 minutes of stirring, tetraethyl orthosilicate $(4.4 \mathrm{~g}, 21 \mathrm{mmol}$ ) was added and reacted for 20 hours at $40^{\circ} \mathrm{C}$. The milky reaction mixture was then transferred to an autoclave and aged at $100^{\circ} \mathrm{C}$ for 24 hours under static conditions. The mixture was allowed to cool to room temperature, and the white precipitate was isolated by filtration, dried in air for at least 2 days, and calcined at $550^{\circ} \mathrm{C}$ for 6 hours in air to produce the SMC materials.

The SMC/PHBHHx composite scaffolds were prepared by a solvent casting-particulate leaching method, using $\mathrm{NaCl}$ particles as the porogen. The PHBHHx was dispersed in chloroform (20 wt $\%$ ), and SMC powders were added to the solution with different compositions $(0,5,10$, and $20 \mathrm{wt} \%$, PHBHHx base) and continuously stirred for 24 hours to disperse SMC uniformly. $\mathrm{NaCl}$ particles with diameters $300-500 \mu \mathrm{m}$ were then incorporated into the suspension, and the dispersion was cast into a $60 \mathrm{~mm}$ diameter cylindrical PTFE mold. The weight ratio of PHBHHx and SMC to $\mathrm{NaCl}$ was 1:8. The samples were air-dried under flowing air for 24 hours to allow the solvent to evaporate and were subsequently vacuum-dried at $40^{\circ} \mathrm{C}$ for 48 hours to remove any remaining solvent. The samples were then immersed in deionized water for 72 hours. During this period, the water was replaced approximately every 6 hours with fresh water at room temperature to leach the salt particulates. The samples were taken out, air-dried for 24 hours, and vacuum-dried overnight to obtain the sponge-like scaffolds. The PHBHHx and SMC/PHBHHx composite films were similarly prepared.

\section{Characterization of SMC and scaffolds}

The morphology of the SMC powders was analyzed using transmission electron microscope (TEM; JEOL2100; JEOL, Tokyo, Japan), working at $200 \mathrm{kV}$. The $\mathrm{N}_{2}$ adsorptiondesorption isotherms were obtained using a Micromeritics porosimeter (TriStar 3000; Norcross, GA, USA) at $77 \mathrm{~K}$, under 
a continuous adsorption condition. Brunauer-Emmet-Teller and Barrett-Joyner-Halenda analyses were used to determine the surface area, pore size, and pore volume.

The surface morphology of the scaffolds was analyzed by field emission scanning electron microscopy (SEM; Quanta 250; FEI, Hillsboro, OR, USA). To determine the surface hydrophilicity and energy, static contact angle measurements were performed, using distilled water and ethylene glycol as the media, by the sessile drop method on a drop-shape analysis system (JC 2000D3; Shanghai Zhongcheng Digital Technology Co, Ltd, Shanghai, People's Republic of China) at ambient humidity and temperature. Five measurements were performed on different points of each specimen. The surface energy was calculated according to the surface tension and the contact angle.

\section{In vitro bioactivity}

The simulated body fluid (SBF) solution was prepared according to the literature ${ }^{21}\left(\mathrm{SBF}: \mathrm{Na}^{+}, 142.0 ; \mathrm{K}^{+}, 5.0 ; \mathrm{Mg}^{2+}, 1.5\right.$; $\mathrm{Ca}^{2+}, 2.5 ; \mathrm{Cl}^{-}, 147.8 ; \mathrm{HCO}_{3}^{-}, 4.2 ; \mathrm{HPO}_{4}{ }^{2-}, 1.0$; and $\mathrm{SO}_{4}{ }^{2-}$, $0.5 \mathrm{~mol} \mathrm{~m}^{-3}$ ). Three samples of each scaffold type were soaked in $50 \mathrm{~mL} \mathrm{SBF}$ at a temperature of $37^{\circ} \mathrm{C}$ while shaking at $100 \mathrm{rpm}$. After 28 days, the specimens were removed and gently rinsed with distilled water and dried overnight at a temperature of $60^{\circ} \mathrm{C}$. The samples were then sputter-coated with gold, and the presence and morphology of apatite formed on the material surface were observed, using field emission SEM.

\section{Adsorption and releasing assay}

To absorb the recombinant human bone morphogenetic protein (rhBMP-2) on the surfaces of the scaffolds, the scaffolds were immersed in $5 \mathrm{~mL}$ rhBMP-2 solutions $(100 \mu \mathrm{g} / \mathrm{mL})$ at room temperature for 72 hours with gentle shaking. Then the scaffolds were washed three times with phosphate-buffered saline (PBS; $\mathrm{pH} 7.4$ ) and dried in a vacuum at room temperature for 48 hours. The loading efficiency of rhBMP-2 onto the scaffolds was calculated by subtracting the amount of rhBMP-2 left in the solution from the amount of rhBMP-2 in the control PBS solution that was treated under the identical incubation process. To investigate the release behavior of the growth factor, the rhBMP-2-adsorbed scaffolds were incubated in $2 \mathrm{~mL}$ PBS supplemented with $1 \%$ bovine serum albumin (Sigma-Aldrich) at $37^{\circ} \mathrm{C}$ for up to 14 days with mild shaking (50 rpm). The media were collected and replaced with fresh PBS periodically, and the rhBMP-2 in the collected medium was determined using an enzyme-linked immunosorbent assay kit (BioVision, CA, USA) according to the manufacturer's protocol. Light absorbance was read with a microreader at $450 \mathrm{~nm}$.

\section{Cell culture}

The human mesenchymal stem cells (hMSCs) were cultured as described in a previous study. ${ }^{22}$ In brief, cells were cultured in $\alpha$-MEM (minimum essential medium, alpha) culture medium supplemented with $10 \%$ fetal bovine serum, $1 \%$ penicillin $(100 \mathrm{U} / \mathrm{mL})$, and streptomycin sulphate $(100 \mathrm{mg} /$ $\mathrm{mL}$; Life Technologies, Carlsbad, CA, USA). The cells were incubated at $37^{\circ} \mathrm{C}$ in a humidified atmosphere of $5 \% \mathrm{CO}_{2}$ and 95\% air with the growth medium changed every 48 hours. hMSCs at passage 3 were detached by $0.25 \%$ trypsin, resuspended in a different density using fresh culture medium, and used for the experiments described here.

\section{Cell adhesion and proliferation}

A single milliliter of the cell suspension with cell density of $2 \times 10^{4}$ viable cells was seeded in a 48 -well plate (Costar3548; Corning Life Sciences, Corning, NY, USA) that contained four different kinds of the scaffolds and was incubated at $37^{\circ} \mathrm{C}$ in a humidified atmosphere of $5 \% \mathrm{CO}_{2}$ and $95 \%$ air for 6 hours. At a 6 -hour time point, cells on the surface of the four kinds of scaffolds were stained with $4^{\prime}, 6^{\prime}$-diamidino-2-phenylindole (DAPI). The cells were observed using a fluorescence microscope (TE2000U; Nikon Corporation, Tokyo, Japan).

The proliferation of the hMSCs was examined with a 3-(4, 5-dimethylthiazol-2-yl)-2, 5-diphenyltetrazolium bromide (MTT) assay after 1, 4, and 7 days of culture. At the prescribed time, $0.1 \mathrm{~mL}$ MTT solution was added, and the specimens were incubated at $37^{\circ} \mathrm{C}$ for 4 hours to form formazan, which was then dissolved using dimethyl sulfoxide. The optical density was measured at $570 \mathrm{~nm}$, using an automated plate reader (Synergy HT multidetection microplate; Biotek, Winooski, VT, USA). The mean absorbance obtained from the medium-control well was determined from the test absorbance values.

\section{Cell morphology}

The cell morphology of the hMSCs was studied by staining the filamentous actin of the cytoskeleton and confocal laser scanning microscopy (Nikon A1R; Nikon, Tokyo, Japan). After 24 hours of incubation with the substrates, as described earlier, the cells on the surface of the substrates were washed gently with PBS three times and then fixed using $3.7 \%$ formaldehyde for 15 minutes at room temperature. The cells were permeabilized with $0.1 \%$ Triton X-100 in PBS for another 15 minutes. After three washings with PBS, the cells were incubated with fluorescein isothiocyanate-Phalloidin (Sigma-Aldrich) for 1 hour. After washing with PBS again, the cell nuclei were stained with DAPI. The cell morphology 
and spreading were visualized using confocal laser scanning microscopy.

\section{Osteogenic differentiation of hMSCs}

hMSCs with a density of $3 \times 10^{4}$ cells $/ \mathrm{mL}$ were used to evaluate the effect of the PHBHHx-based scaffolds on the osteogenic differentiation. After incubation in a 48-well plate with four substrates for 24 hours, the culture medium was changed to osteogenic induction medium: the $\alpha$-MEM culture medium was supplemented with $10 \%$ fetal bovine serum, $0.1 \mu \mathrm{M}$ dexamethasone (Sigma-Aldrich), $50 \mu \mathrm{M}$ ascorbate acid (Sigma-Aldrich), and $10 \mathrm{mM}$ ß-glycerophosphate sodium (Sigma-Aldrich). These media were renewed every 2 days throughout the study.

After 3, 7, and 14 days' culture with the above osteogenic induction medium in a 48-well plate, the alkaline phosphatase (ALP) activity was determined using p-nitrophenyl phosphate (Sigma-Aldrich) as the substrate, and the total protein content was determined according to the procedures as mentioned in a previous article. ${ }^{23}$ Briefly, the ALP activities were determined by a colorimetric assay using an ALP reagent containing p-nitrophenyl phosphate as the substrate. The absorbance of p-nitrophenol that was formed was measured at a wavelength of $405 \mathrm{~nm}$. The intracellular total protein content was determined using the MicroBCA protein assay kit, and the ALP activity was normalized to the total protein content (Biyuntian, Shanghai, People's Republic of China). After 14 days of culture, the ALP staining was accomplished following the procedure in the literature. ${ }^{25}$ In brief, the cells on the scaffolds cultured in the 48-well plate were detached with $100 \mu \mathrm{L}$ of a $0.25 \%$ trypsin solution and then removed to another 48-well plate containing $1 \mathrm{~mL}$ culture media to allow the cells to adhere. After 24 hours, the cells were spread on the bottom of the well, rinsed twice with PBS, and then fixed using buffered formalin for 30 seconds, according to the procedures provided by the manufacturers of the ALP staining kit (Renbao, Shanghai, People's Republic of China). After washing with distilled water twice, the cells were treated with a staining reagent for 60 minutes. Stained cells were photographed using a microscope (TE2000U; Nikon, Tokyo, Japan).

\section{In vivo bone formation}

Four kinds of scaffolds were prepared for this study: pure PHBHHx scaffolds, pure PHBHHx scaffolds loaded with rhBMP-2, PHBHHx-20 scaffolds, and PHBHHx-20 scaffolds loaded with rhBMP-2. Operative procedures and animal care were performed according to the Institutional Guidance of Shanghai 9th People's Hospital on Animal Experimentation. Surgery on the 3-month-old male
New Zealand white rabbits was under standard sterile conditions. Briefly, the rabbits were anesthetized with an intraperitoneal injection of pentobarbital sodium solution (40 mg/kg body weight). After shaving the rabbits' fur and disinfection with 75 vol\% ethanol, a longitudinal incision was made along the forearm skin of the rabbits. The periosteum was incised circumferentially to approach to the radius bone. A critical defect of $15 \mathrm{~mm}$ in length was then created at the middle position of the radius bone by using a side-cutting diamond disk and a high-speed micromotor under abundant irrigation with sterile saline solution. The scaffold was implanted into the defect, and the periosteum and overlying muscle were repositioned with an absorbable polydioxanone suture (Ethicon 5-0; Johnson and Johnson, New Brunswick, NJ, USA). Then, the wound was closed with a nonabsorbable polypropylene suture (Ethicon 5-0). The experiment was independently performed for 4 samples per experimental group at each sampling point.

\section{Micro-computed tomography and histological analysis}

At 4, 8, and 12 weeks postoperatively, the rabbits were killed and the implants were excised and fixed in a $1.5 \%$ phosphate buffered glutaraldehyde solution. The implants were scanned using a micro-computed tomography $(\mu \mathrm{CT})$ system (X-RAY CT System, SMX-100CT-SV3 TYPE; Shimadzu Ltd, Kyoto, Japan) at $0.49^{\circ}$ angular increments, which provided 721 views around $360^{\circ}$. Images were recorded, digitized, and transferred to a controlling computer for reconstruction using a modified Feldkamp cone beam tomographic reconstruction algorithm. The $3 \mathrm{D}$ images consisted of $20-\mathrm{mm}$ cubic voxels, and the radiopacity of each voxel was represented by a 16-bit grayscale value. Image analysis was performed using the Analyze software package (Biomedical Imaging Resource; Mayo Clinic, Rochester, MN, USA). After $\mu \mathrm{CT}$ analysis, the implants were dehydrated in a graded series of alcohol and embedded in methylmethacrylate for histological analysis. Sections were stained with Masson trichrome and hematoxylin and eosin staining for evaluation of the general tissue response and bone formation.

\section{Statistical analysis}

All quantitative data were expressed as the mean \pm standard deviation. Statistical analysis was performed with one-way analysis of variance, using SPSS software (IBM Corporation, Armonk, NY, USA). A value of $P<0.05$ was considered statistically significant. 


\section{Results}

\section{Characterization of SMC and $\mathrm{PHBHH} / \mathrm{SMC}$ scaffold}

Figure 1 shows a collection of representative TEM images and Brunauer-Emmet-Teller analysis of synthesized SMC. The sample possesses a disordered array of silica struts with relatively uniform pore sizes (about $20 \mathrm{~nm}$ ). The porosity of the SMC has been investigated by nitrogen sorption analyses.

The isotherms are of type 4 and show steep hystereses of type $\mathrm{H} 1$ at high relative pressures (see Figure 1C), which is typical for mesoporous materials that exhibit capillary condensation and evaporation and have large pore sizes with narrow size distributions. The surface area and pore volume of SMC are about $575 \mathrm{~m}^{2} / \mathrm{g}$ and $1.4 \mathrm{~cm}^{3} / \mathrm{g}$, respectively.

\section{Physical and chemical properties of composite scaffolds}

Bone tissue regeneration depends not only on the porosity of the scaffolds but also on the pore size and interconnection between the pores. In general, a high porosity with a high interconnection between the pores is necessary to allow ingrowth of cells, vascularization, and diffusion of nutrients and wastes. ${ }^{22,24} \mathrm{SMC} / \mathrm{PHBHHx}$ composite scaffolds were prepared by combining PHBHHx with SMC, using the porogen leaching method. To observe the morphology of scaffolds and distribution of SMC in the polymer matrix of the scaffolds, SEM micrographs at higher magnifications were obtained.
Figure 2 shows the SEM micrographs of the cross-sections of pure PHBHHx scaffolds and SMC/PHBHHx scaffolds containing 5, 10, and $20 \mathrm{wt} \%$ SMC. All the scaffolds have an interconnected porous architecture with pore sizes ranging from 200 to $400 \mu \mathrm{m}$. The SMC content has no significant effect on pore size and interconnection between the pores of the scaffolds. The SMC particles are homogeneously dispersed as primary particles in the polymer matrix of the scaffolds.

Figure $3 \mathrm{~A}$ shows that the contact angle of the PHBHHx-20 against water $\left(36.40^{\circ} \pm 3.62^{\circ}\right)$ was relatively smaller than that of PHBHHx-10, PHBHHx-5, and PHBHHx (56.20 $\pm 5.32^{\circ}$, $62.34^{\circ} \pm 4.65^{\circ}$, and $89.62^{\circ} \pm 4.48^{\circ}$, respectively; $P<0.05$ ). Another index to evaluate the hydrophilicity is the increase of the surface energy, and Figure 3A shows that the surface energy of PHBHHx-20 was higher than that of the other three specimens $(58.72 \pm 4.87,48.27 \pm 4.01,43.21 \pm 3.97$, and $37.84 \pm 1.89 \mathrm{~J} / \mathrm{m}^{2}$ for PHBHHx-20, PHBHHx-10, PHBHHx-5, and PHBHHx, respectively; $P<0.05$ ). Representative images of the water contact angles are shown in Figure 3B, and it can be seen that the radian of the water drop on PHBHHx-20 was dramatically smaller than that of the other three specimens.

\section{rhBMP-2 adsorption and release}

BMP-2 amount on 3D scaffolds was first measured by the direct enzyme-linked immunosorbent assay. Figure 4A shows the amount of rhBMP-2 adsorption on the surface
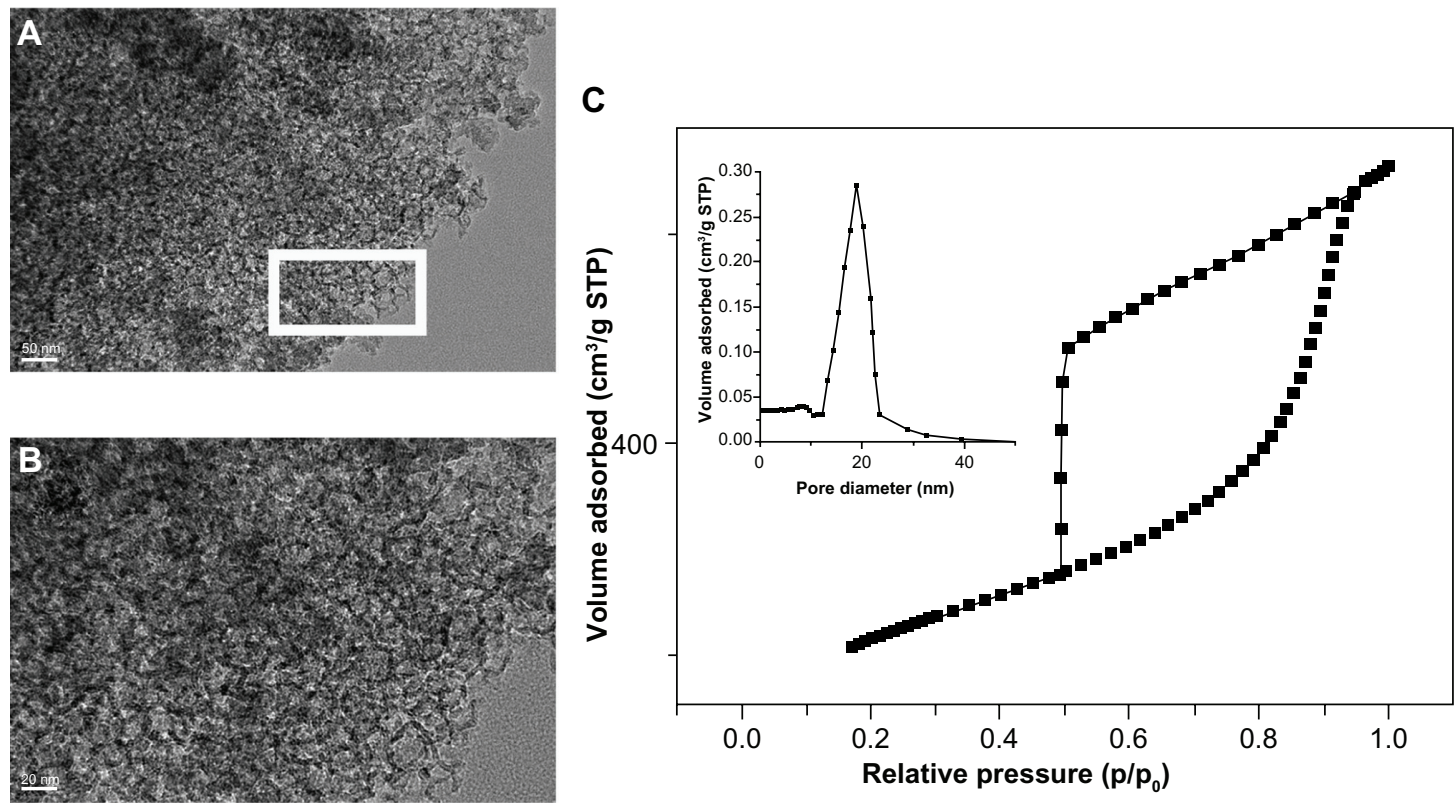

Figure I Transmission electron microscope images (A and B) and Brunauer-Emmet-Teller analysis (C) of pore siliceous mesostructured cellular foams. (B) Enlarged images of panel A. Transmission electron microscope image of siliceous mesostructured cellular foam powders show a disordered array of silica struts with relatively uniform pore sizes (about $20 \mathrm{~nm}$ ).

Abbreviation: STP, standard temperature and pressure. 


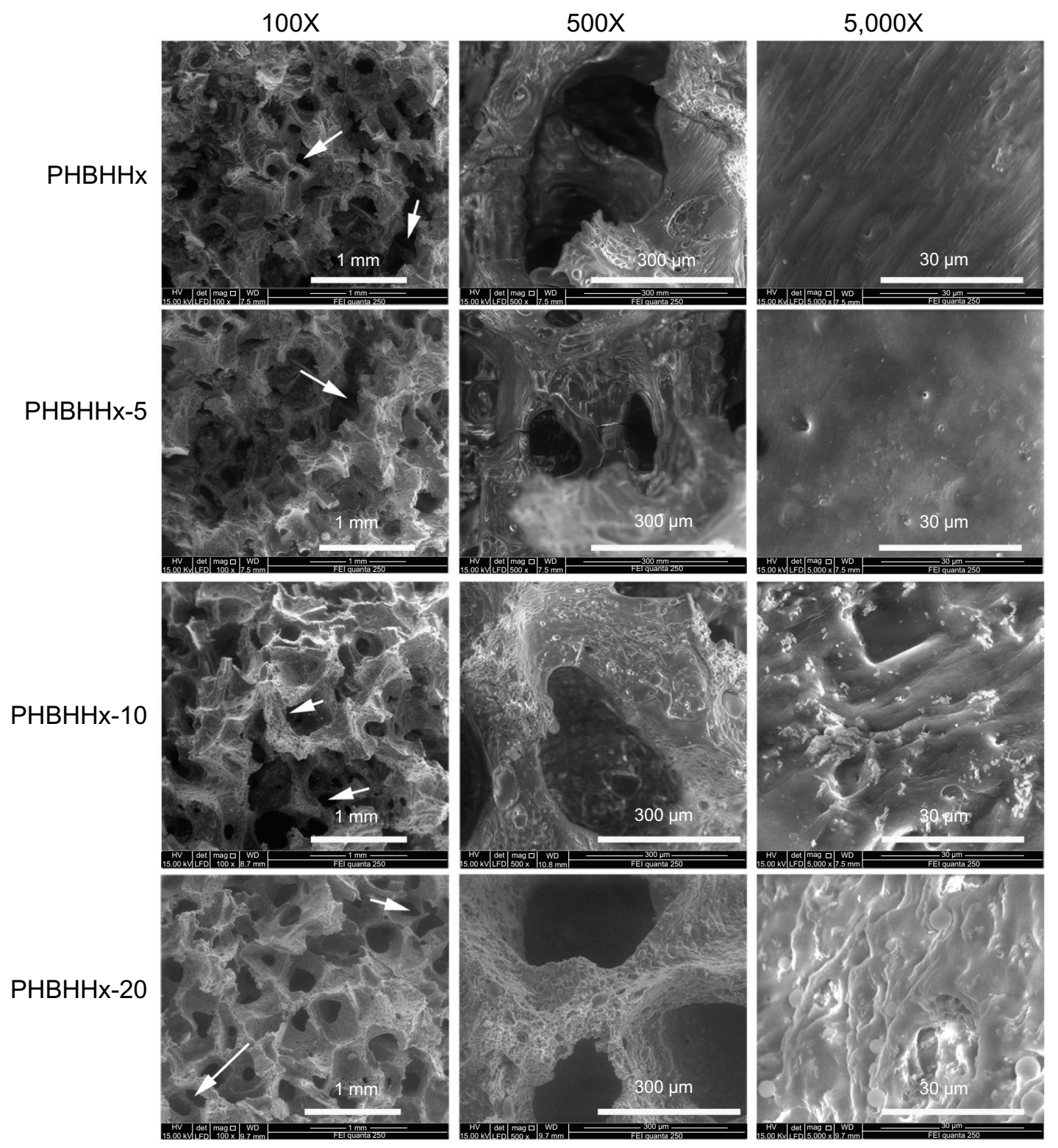

Figure 2 Scanning electron microscope morphology of pure poly(3-hydroxybutyrate-co-3-hydroxyhexanoate) (PHBHHx) and siliceous mesostructured cellular foam/poly(3hydroxybutyrate-co-3-hydroxyhexanoate) composite three-dimensional scaffold. The siliceous mesostructured cellular foam content has no significant effect on pore size and interconnection between the pores of the scaffolds (white arrows).

of the porous SMC/PHBHHx scaffolds with different SMC compositions (0-20 wt\%), as determined by the enzymelinked immunosorbent assay. The amount of rhBMP-2 immobilized on the scaffolds increases with increasing SMC composition (PHBHHx, 8.65\% $\pm 2.36 \%$; PHBHHx-5, $17.39 \% \pm 4.68 \%$; PHBHHx-10, $46.98 \% \pm 8.25 \%$; and PHBHHx-20, 75.63\% $\pm 10.65 \%$ ), as expected.

The sustained release patterns of rhBMP-2 are shown in Figure 4B. The four scaffolds had similar release behavior during the observation period, which is a two-step release behavior: an initial burst release followed by a relatively slow release.
For the PHBHHx scaffold, an initial burst release in the first 5 days is around $79 \%$ of the total amount of rhBMP-2. The subsequent release rate remarkably decreased as a function of time, and the cumulative release reaches a maximum value of $94 \%$ at 10 days. After the addition of SMC, around $24 \%, 35 \%$, and $47 \%$ of the total rhBMP-2 loading is released in the first 5 days for PHBHHx-5, PHBHHx-10, and PHBHHx-20, respectively. After 27 days, the amount of released rhBMP-2 reaches $56 \%, 69 \%$, and $82 \%$ for PHBHHx-5, PHBHHx-10, and PHBHHx-20. Therefore, the rhBMP-2 release rate of the SMC/PHBHHx scaffolds is significantly lower than that of the PHBHHx scaffold. 


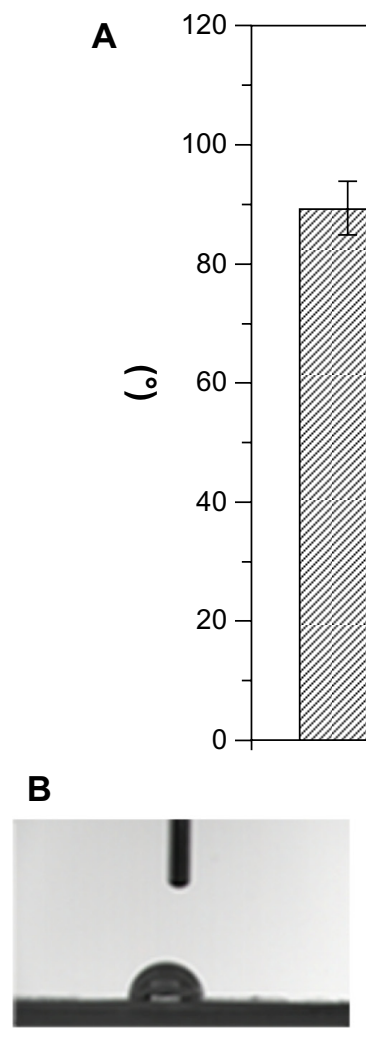

PHBHHx

\section{B}

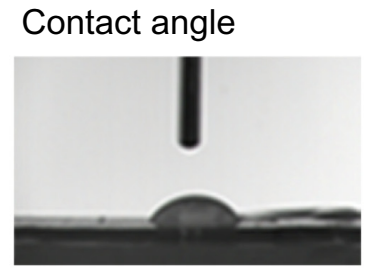

PHBHHx-5

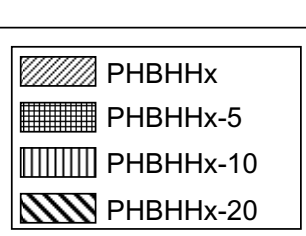

120

100

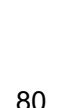

80

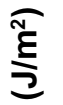

Figure 3 Water contact angles and surface energy measured on the surface of the samples (A). Representative images of the water contact angles (B). Abbreviation: $\mathrm{PHBHHx}$, poly(3-hydroxybutyrate-co-3-hydroxyhexanoate).

For the SMC/PHBHHx scaffolds, rhBMP-2 adsorbed on the surface and at the window of mesoporous channels is quickly released at early times, resulting in the initial burst release. Although some of rhBMP-2 is hosted inside the mesoporous channels, this release must diffuse through the channels. Moreover, the rhBMP-2 molecules contain hydroxyl groups and amino groups, which could interact with the $\mathrm{Si}-\mathrm{OH}$ groups in the SMC by hydrogen bonding to slow the release. The rhBMP-2 detected on the pure PHBHHx scaffolds can be also explained by the adsorption of rhBMP-2 on the pore surfaces of the scaffolds, which may lead to an initial burst release in the medium. The sustained release
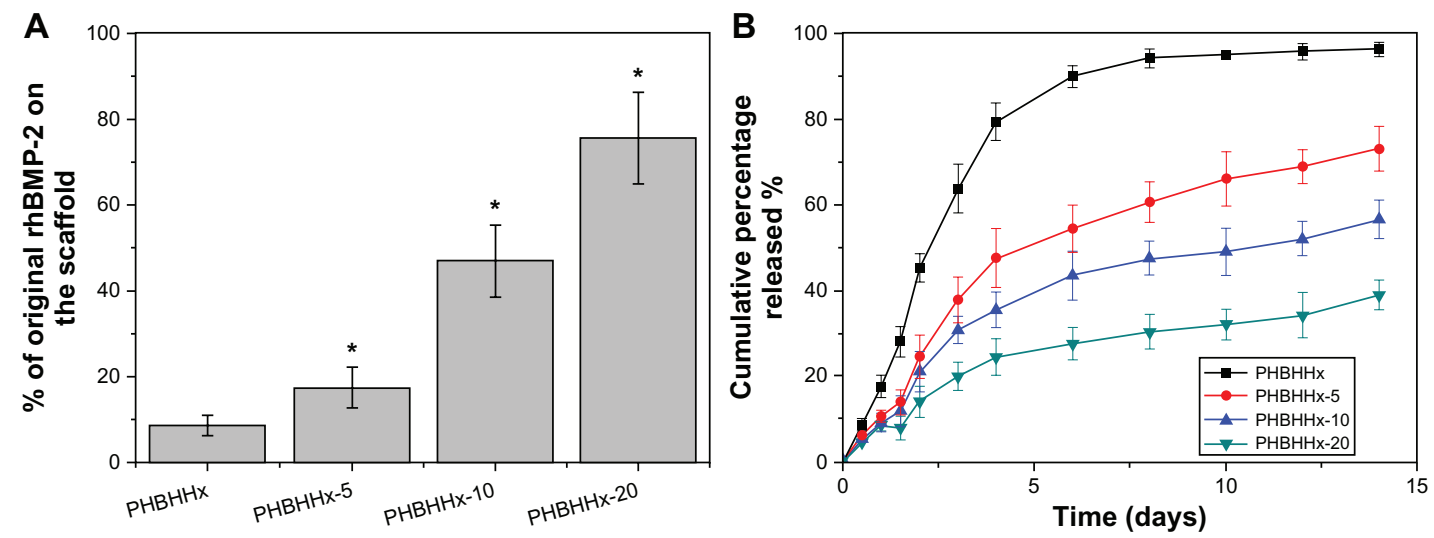

Figure 4 (A) Adsorption of recombinant human bone morphogenetic protein 2 amounts on the poly(3-hydroxybutyrate-co-3-hydroxyhexanoate) (PHBHHx) and siliceous mesostructured cellular foam/PHBHHx scaffolds. The amount of bone morphogenetic protein 2 remaining on the scaffolds was present as the difference between the original amount and the amount in the wash buffer. The values represent the percentage of the remaining amount on the scaffolds of the original loaded amount ( $\mathrm{n}=4$ ). (B) Profiles of recombinant human bone morphogenetic protein 2 release from $\mathrm{PHBHHx}$ and SMC/PHBHHx scaffolds with $20 \mu \mathrm{g}$ recombinant human bone morphogenetic protein 2 adsorption. The amount of recombinant human bone morphogenetic protein 2 released from scaffolds was determined by enzyme-linked immunosorbent assay. The values represent the mean \pm standard error $(n=4)$. $* P<0.05$ compared with the $\mathrm{PHBHHx}$ group. 
of rhBMP-2 from the scaffolds may be very helpful for the acceleration of bone regeneration through the scaffolds, and thus facilitate effective regeneration of bone with sufficient mechanical properties.

\section{In vitro bioactivity}

The test of the acellular bioactive behavior of scaffolds was performed in vitro through the immersion of samples in SBF. Figure 5 shows the surface of the four scaffolds after immersion in SBF for 28 days. It is evident that many ball-like particles formed on the surfaces of PHBHHx-20 and PHBHHx-10, predominantly on the $\mathrm{PHBHHx}-20$ surface. More apatite particles formed as SMC increased. The apatite layer is quite dense on the PHBHHx-20 scaffolds (Figure 5D1-D3), but only a few apatite particles deposit on the surface of the pure PHBHHx scaffold (Figure 5A1-A3).

\section{Cellular affinity}

\section{Cell attachment and proliferation}

In the present study, DAPI staining was used to observe cell attachment, and the MTT assay was performed to evaluate the proliferation of hMSCs cultured on PHBHHx or SMC/ PHBHHx scaffolds. The number of adherent cells on the four specimen surfaces stained with DAPI are shown in Figure 6A. Cell numbers increased with increasing SMC content. Cell proliferation values on the samples after 4 and 7 days were
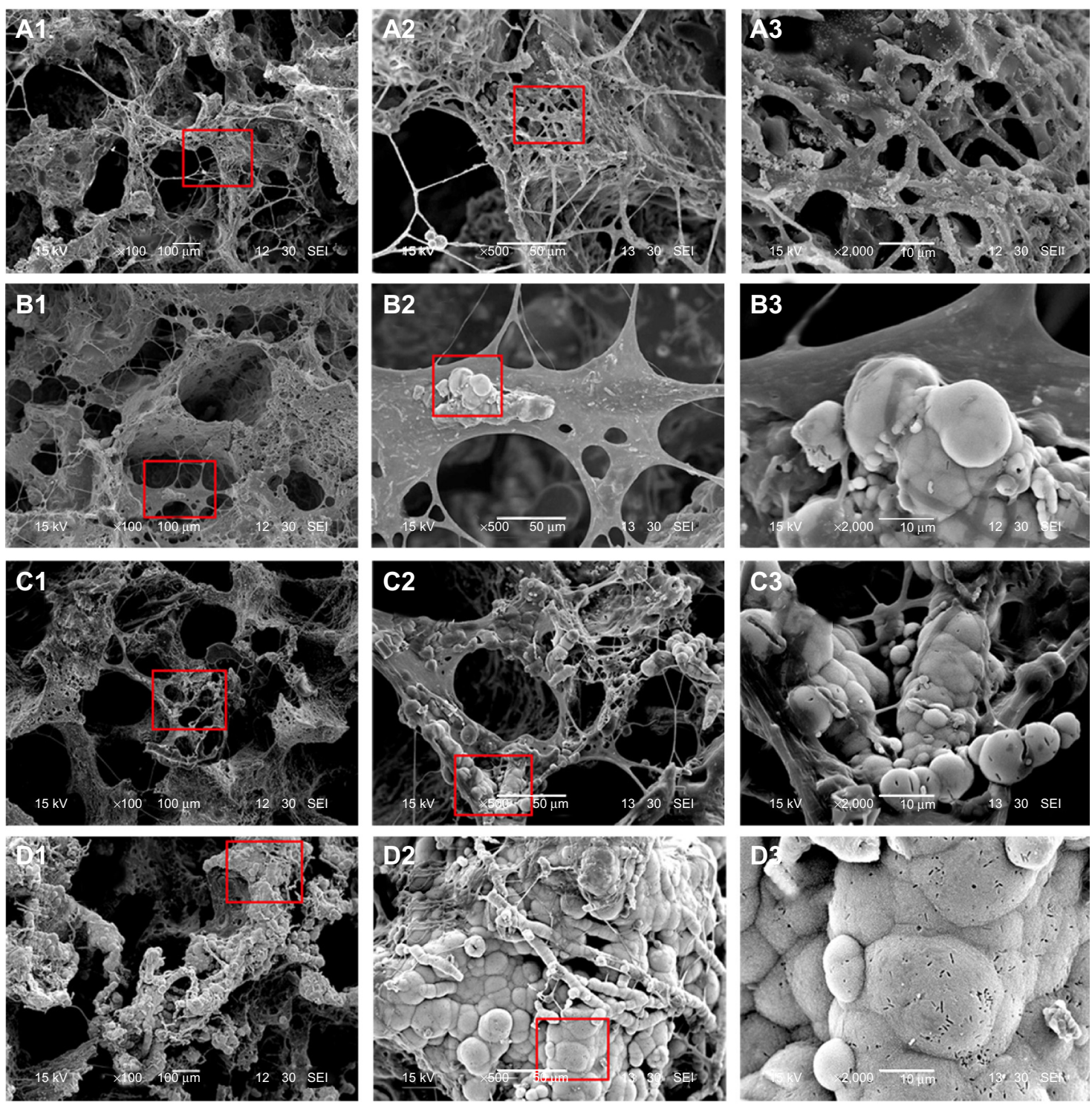

Figure 5 Scanning electron microscope imaging of the scaffolds after immersion in simulated body fluid for 28 days. No obvious changes were evident on the surface of poly(3-hydroxybutyrate-co-3-hydroxyhexanoate) ( $\mathrm{PHBHHx}$ ) (AI-A3). A few particles formed on the PHBHHx-5 surface (B I-B3). Many ball-like particles formed on the surface of $\mathrm{PHBHHx}-10$ and $\mathrm{PHBHHx}-20$ (CI-C3 and DI-D3 respectively). Red square represents magnification area. 
A
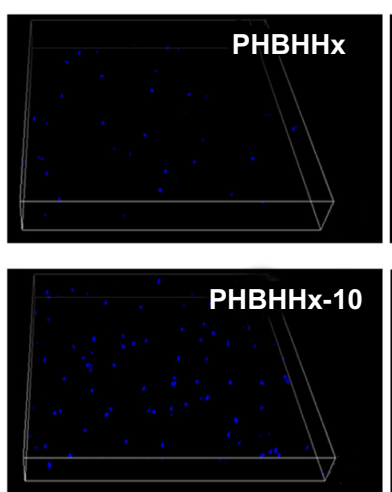
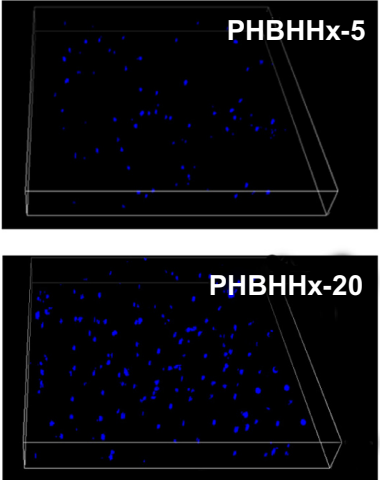

B

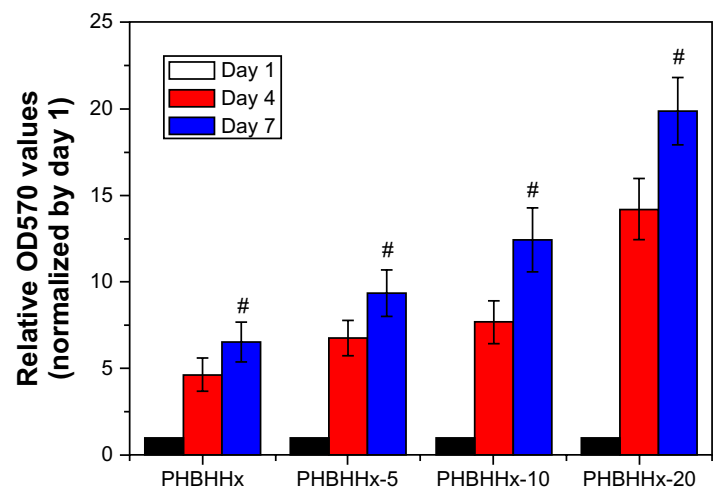

Figure 6 Attachment of the human mesenchymal stem cells on the different surfaces of the scaffolds. (A) Cells stained with $4^{\prime}, 6^{\prime}$-diamidino-2-phenylindole after 6 hours of incubation. (B) The proliferative tendency of the human mesenchymal stem cells from day I to day 7. Compared with day 4 , ${ }^{P}<<0.05$.

Abbreviations: $\mathrm{PHBHHx}$, poly(3-hydroxybutyrate-co-3-hydroxyhexanoate); $\mathrm{OD}$, optical density.

normalized to that of day 1 because the cell numbers were different from 1 day after seeding. Compared with the other specimens at different times, cells on PHBHHx-20 showed an obvious relative proliferation rate $(P<0.05$; Figure $6 \mathrm{~B})$. Compared with PHBHHx-20 at day 4, the cells on PHBHHx-5 and PHBHHx-10 exhibited a lower proliferation rate $(P<0.05$; Figure $6 \mathrm{~B})$. The relative proliferation rate of the hMSCs on the PHBHHx and SMC/PHBHHx increased from day 1 to day 7 .

\section{Cell morphology}

Cell adhesion is one of the most important parameters that control cell-biomaterial interactions. PHBHHx is highly hydrophobic and supports low levels of cell adhesion. Figure 7 shows the cells spreading on the surface of PHBHHx and SMC/PHBHHx. They exhibited a polygonal and fusiform-shaped morphology. The cells on the PHBHHx-20
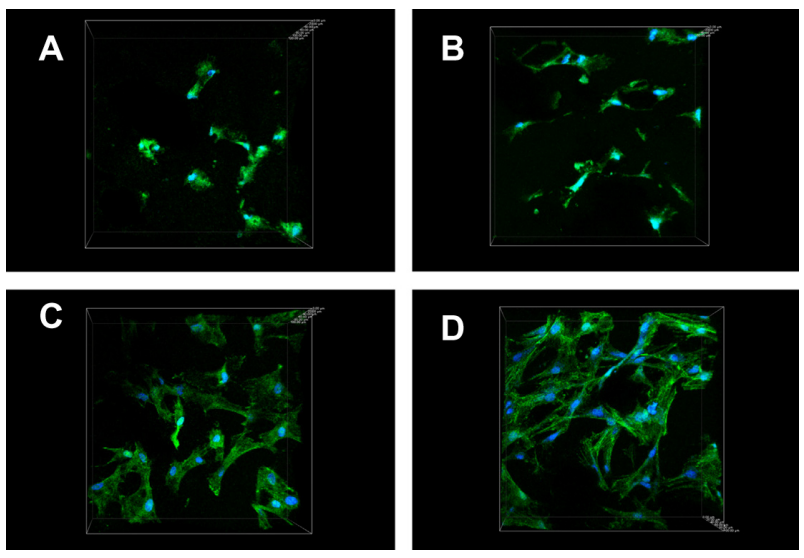

Figure 7 The cytoskeletal morphology and spreading of the human mesenchymal stem cells on the different scaffolds. (A) Poly(3-hydroxybutyrate-co-3-hydroxyhexanoate) (PHBHHx). (B) PHBHHx-5. (C) PHBHHx-10. (D) PHBHHx-20. Representative images of cells stained with fluorescein isothiocyanate-phalloidin for actin filaments (green) and nuclei counterstained with 4',6'-diamidino-2-phenylindole (blue). surface also exhibited clustering and confluency morphology. In addition, the cell numbers on the PHBHHx-20 surface were higher than those on the other specimen surfaces.

\section{Differentiation of hMSCs on the surface of specimens}

Osteogenic differentiation of hMSCs on the scaffolds was assessed by alkaline phosphatase activity. Figure $8 \mathrm{~A}$ shows positive ALP staining on the PHBHHx and SMC/PHBHHx at day 14 and that the staining on the PHBHHx-20 surfaces was significantly denser than that on the PHBHHx surface. In Figure 8B, the hMSCs on the surface of PHBHHx-20, PHBHHx-10, and PHBHHx-5 show significantly higher ALP activity than those cultured on the PHBHHx surface at day 7 and day $14(P<0.01)$. No differences were observed between PHBHHx-10 and PHBHHx-5. By the 14th day, the ALP activity of the hMSCs on the SMC/PHBHHx surfaces increased and no differences were found $(P>0.05)$. Notably, the hMSCs cultivated for 3, 7, and 14 days on PHBHHx had the lowest ALP activity level compared with those on SMC/ PHBHHx $(P<0.05)$.

\section{In vivo animal study}

The 3D restructured images were conducted with a $\mu \mathrm{CT}$ system at 4, 8 and 12 weeks to investigate the in vivo osteoinductivity of PHBHHx and PHBHHx-20 scaffolds incorporating bioactive molecules (Figure 9). The results showed that group A had the least bone binding and slowest healing rate, with no noteworthy bone formation until 8 weeks. The effect of group B was comparatively better than group A in repair rate and integrity but was still imperfect. Both groups $\mathrm{C}$ and D exhibited comparatively good bridging and fast healing. Group $\mathrm{D}$ performed slightly better than group $\mathrm{C}$. The regenerated bone mineral content (BMC) and bone mineral density 
A

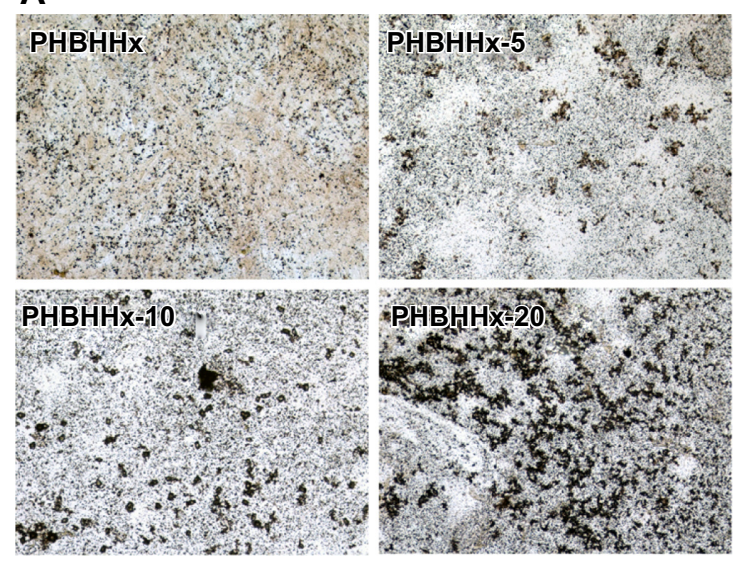

B

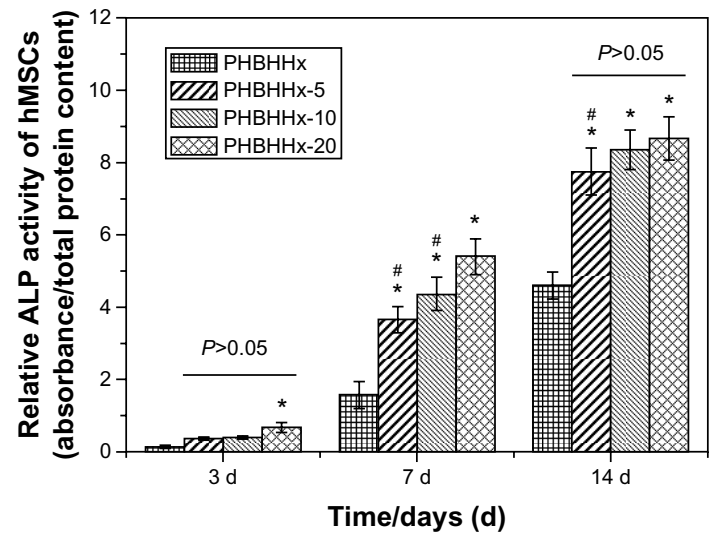

Figure 8 The alkaline phosphatase (ALP) activity and ALP staining assay. (A) Positive ALP staining on the four scaffolds at day I4. The staining on the poly(3-hydroxybutyrateco-3-hydroxyhexanoate)-20 ( $\mathrm{PHBHHx}-20$ ), $\mathrm{PHBHHx}-10$, and $\mathrm{PHBHHx}-5$ surfaces was significantly denser than that on the PHBHHx surface. (B) Relative ALP activity of human mesenchymal stem cells after 6,10 , and I 4 days of culture. An asterisk denotes a significantly higher ALP activity of cells than that on the $\mathrm{PHBHHx}$ surface $(P<0.0 \mathrm{I})$. A pound sign denotes a significantly lower ALP activity level compared with that on PHBHHx-20 at day 7 and day $14(P<0.05)$.

$4 \mathrm{w}$

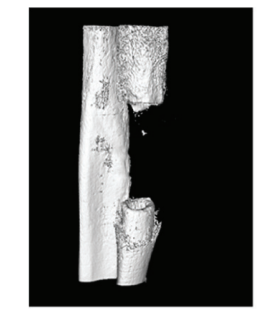

B

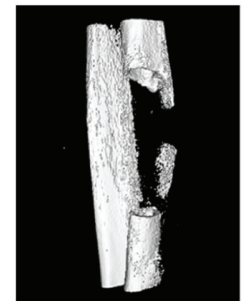

C

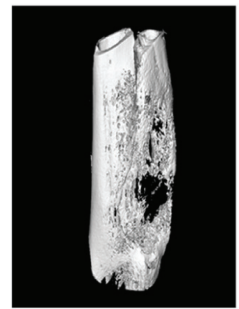

D

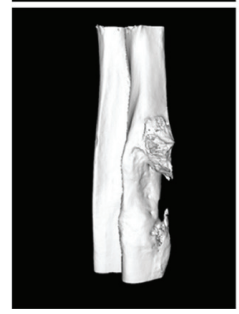

$8 w$
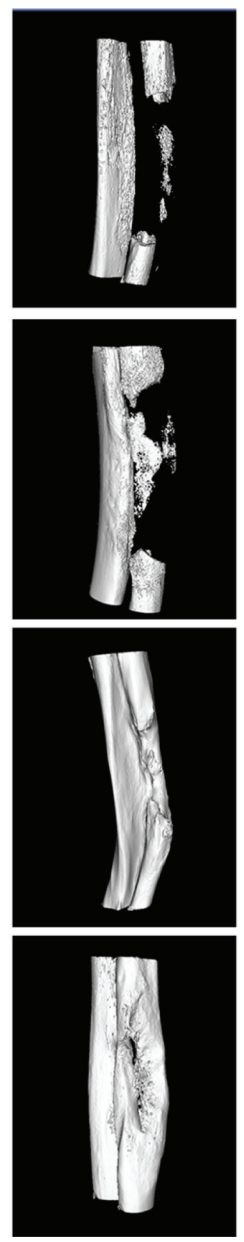

12 w
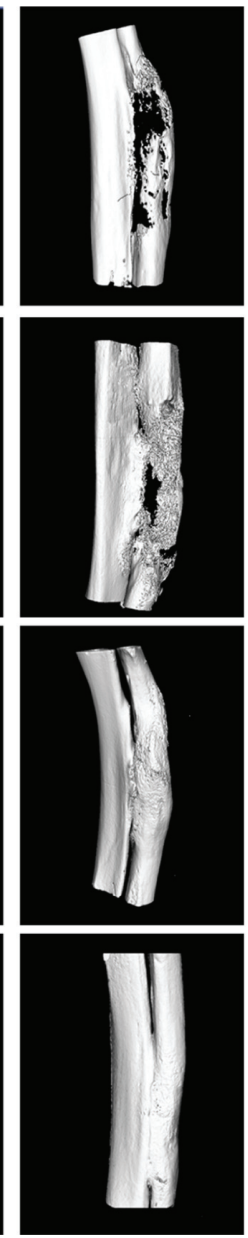

Figure 9 Micro-computed tomography images of segmental radius at 4, 8, and 12 weeks (w) with different implants. Column I-3, Three-dimensional reconstructed images. (A) Pure poly(3-hydroxybutyrate-co-3-hydroxyhexanoate) (PHBHHx) scaffold. (B) PHBHHx-20 scaffold. (C) Recombinant human bone morphogenetic protein 2-loaded PHBHHx scaffold. (D) Recombinant human bone morphogenetic protein loaded $\mathrm{PHBHHx}-20$ scaffold. of the defect area were calculated to quantitatively analyze the repair more precisely (Figure 10). An increase in BMC values was observed for all groups, among which group $\mathrm{C}$ and $\mathrm{D}$ had much higher BMC than groups $\mathrm{A}$ and $\mathrm{B}$ at each point, confirming the results of $3 \mathrm{D}$ images. The bone mineral density values in Figure 10 implied a similar profile, with the lowest being found in group $\mathrm{A}$ and the highest in group D.

To detect more detailed repair processes, including various soft tissue growths that could not be discerned with $\mu \mathrm{CT}$ scanning, we used histology including hematoxylin and eosin and Masson trichrome staining. Figure 11 provides integral imaging on the finally formed medullary cavity of radius. The medullary cavity for the self-repairing group and pure PHBHHx scaffolds group failed in reunion (Figure 11A1-A4 and 11B1-B4), whereas the rhBMP-2-loaded PHBHHx scaffolds and rhBMP-2-loaded PHBHHx/SMC scaffolds succeeded in repair and healing by forming mature cortical bone and complete marrow cavity that presented both erythrocytes medulla ossium rubra and medulla ossium flava. Specifically, group D slightly surpassed group C in the integrity of peripheral cortical bone (Figure 11D1-D4).

\section{Discussion}

Creating novel scaffolds for bone regeneration is an essential area for planning tissue engineering approaches. ${ }^{25}$ Mesoporous materials have been extensively studied as bone implant materials because of their osteoinductive properties and biocompatibility. ${ }^{26}$ However, mesoporous materials are highly brittle and of low toughness, which restricts their widespread use as ideal scaffolds. ${ }^{27}$ Synthetic bioresorbable polymers are easily fabricated into complex structures 

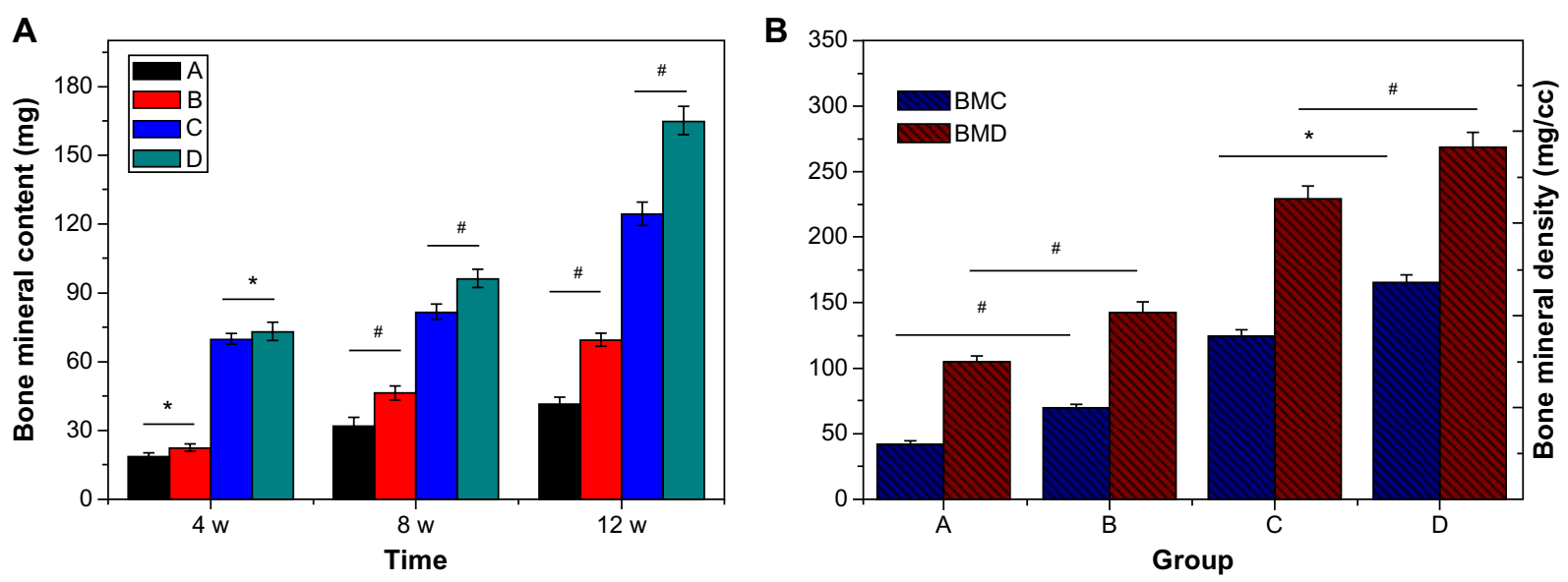

Figure 10 Quantitative data of orthotopic bone formation with different implants. (A) Bone mineral content (BMC) at 4, 8, and I2 weeks (w). (B) BMC and bone mineral density (BMD) at 8 weeks. Group A: Self-repairing without any implants. Group B: Pure poly(3-hydroxybutyrate-co-3-hydroxyhexanoate) (PHBHHx) scaffold. Group C: Recombinant human bone morphogenetic protein 2-loaded PHBHHx scaffold. Group D: Recombinant human bone morphogenetic protein-loaded PHBHHx/ siliceous mesostructured cellular foam scaffold. ${ }^{P}>0.05$, no significant differences were found. $* P<0.05$, significant differences were found.

but have low efficiency of cell attachment and proliferation and poor osteoconductivity because they lack specific cellrecognition signals. ${ }^{28}$ To overcome these problems, SMC particles were incorporated into biodegradable PHBHHx matrix materials. The SMC/PHBHHx composite scaffolds with 5\%, $10 \%$, and $20 \%$ SMC have been successfully prepared using a solvent casting and salt-leaching method. The composite scaffolds produced by this method are highly porous and have a structure of well-interconnected pore channels that benefits tissue ingrowth. ${ }^{29}$

The addition of SMC into the PHBHHx matrix significantly improved in vitro apatite mineralization as well as attachment, proliferation, and early differentiation of hMSCs compared with pure PHBHHx. Apatite mineralization is thought to be an important phenomenon in the chemical interactions between the implant materials and the bone
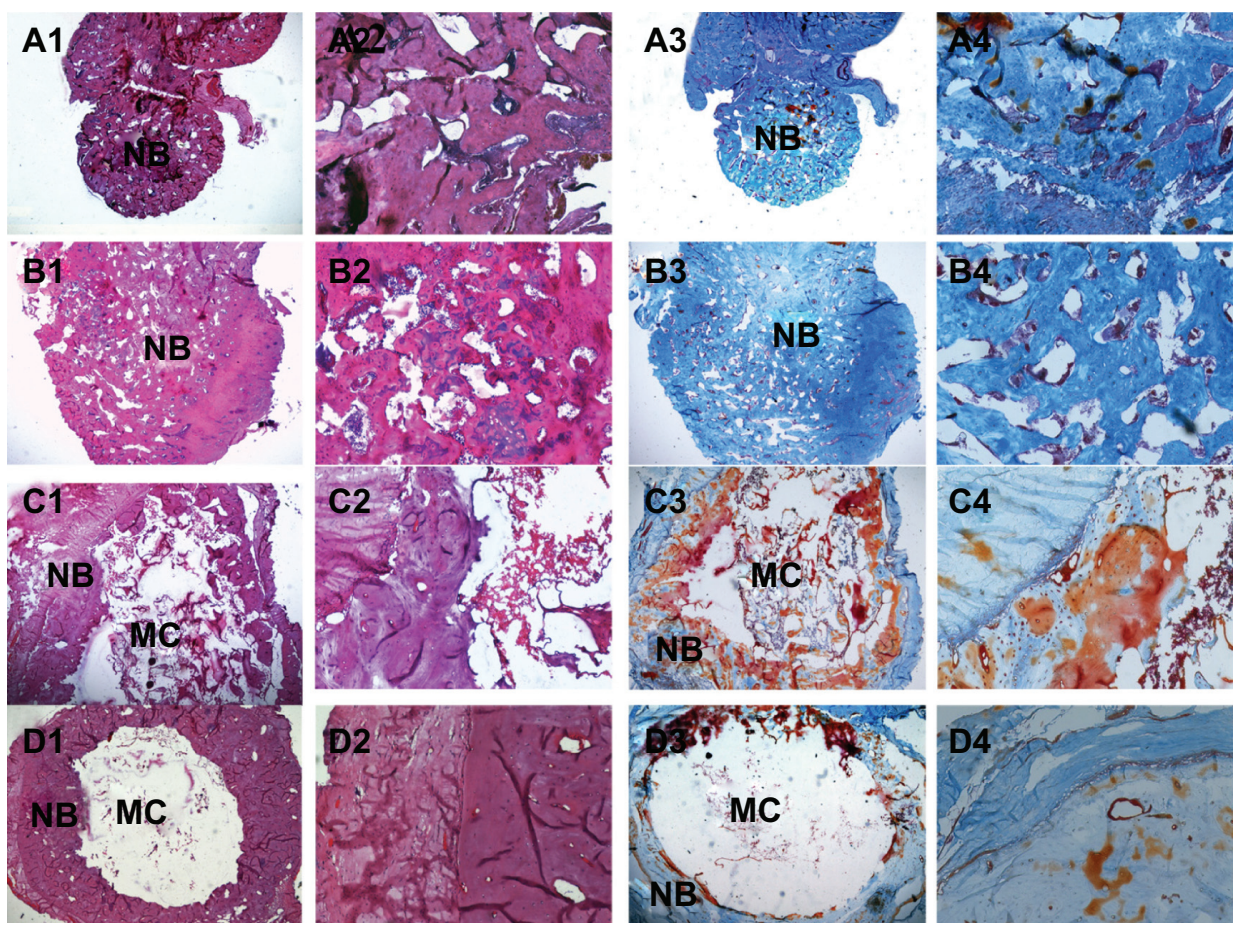

Figure II Histological evaluation of orthotopically formed bone sections at 12 weeks (AI, A2, BI, B2, CI, C2, DI, and D2 are HE staining; A3, A4, B3, B4, C3, C4, D3, and D4 are Masson trichrome staining). (A) Pure poly(3-hydroxybutyrate-co-3-hydroxyhexanoate) (PHBHHx) scaffold. (B) PHBHHx-20 scaffold. (C) Recombinant human bone morphogenetic protein 2-loaded PHBHHx scaffold. (D) Recombinant human bone morphogenetic protein 2-loaded PHBHHx-20 scaffold.

Abbreviations: MC, marrow cavity; NB, new bone; HE, hematoxylin and eosin. 
tissue, which ultimately affects the in vivo osteogenesis of the bone grafting materials. ${ }^{30}$ In this study, SMC/PHBHHx scaffolds showed obvious apatite mineralization in SBF, whereas only a few apatite particles deposit on the surface of the pure PHBHHx scaffold.

$\mathrm{Si}-\mathrm{OH}$ groups had been proposed as a catalyzing agent and could provide specific favorable sites for apatite nucleation. This suggests that the formation of apatite might be closely related to the amount of $\mathrm{Si}-\mathrm{OH}$ groups presented on the surface of scaffolds and that SMC/PHBHHx scaffolds have an improved in vitro bioactivity. Enhanced attachment, proliferation, and early differentiation of hMSCs on the SMC/ PHBHHx may result from the direct contact of seeded cells with the SMC particles exposed on the scaffold surface, which might provide cell adhesion sites with better cellular adherence and spreading to stimulate cell proliferation and osteogenic differentiation. ${ }^{31,32}$

In addition, $\mathrm{SMC} / \mathrm{PHBHHx}$ has a higher surface area than pure PHBHHx matrix; most researchers believe that this may promote cell growth more efficiently than a bulk substrate. We also determined that the percentage of hMSCs attached on the SMC/PHBHHx composite with $20 \mathrm{wt} \% \mathrm{SMC}$ was higher than on pure $\mathrm{PHBHHx}$. The improved hydrophilicity of the MCS/SF composite films might contribute to these results. Thus, SMC acts as anchoring point for cell adhesion and spreading. Together, these data leave little doubt that SMC possesses excellent in vitro bioactivity.

ALP is upregulated at the onset of osteoblastic differentiation and subsides after differentiation progresses; our results suggest that the introduction of SMC into PHBHHx scaffolds may be beneficial for osteogenesis. Previous studies have demonstrated that the addition of millimolar concentrations of calcium ions could enhance the proliferation and phenotype of osteoblast cells by membrane-mediated ion transfer. ${ }^{33,34}$ Calcium ions play an important role in the regulation of osteoblastic proliferation and differentiation by changing the expression levels of specific calcium ion channel isoforms in osteoblasts. ${ }^{35}$

In this study, we also compare the in vivo effect of SMC/ PHBHHx and PHBHHx on osteogenesis by implanting the rhBMP-2 adsorption scaffolds into the radius defects of New Zealand rabbits. The $\mu \mathrm{CT}$ and Van Gieson staining analyses confirmed that all scaffolds showed bone induction after 8 weeks of radius bone implantation, and SMC/PHBHHx scaffolds were superior in all aspects compared with pure PHBHHx scaffolds. Many studies have confirmed that the sustained release of rhBMP-2 is known to be effective in promoting new bone formation. In our study, the release rate of rhBMP-2 can be controlled by SMC content, and the prolonged release can induce mature bone formation.

\section{Conclusion}

The SMC/PHBHHx composite scaffolds with well-defined macropores were successfully prepared by a porogen leaching method. The scaffold showed a good degree of interconnective pore structure in favor of cell seeding and spatial distribution of transplanted cells. The specific areas and pore volume of synthesized SMC are $575 \mathrm{~m}^{2} / \mathrm{g}$ and $1.4 \mathrm{~cm}^{3} / \mathrm{g}$, respectively. The SMC powders have a disordered channel structure with a pore size of approximately $21 \mathrm{~nm}$. The incorporation of SMC into PHBHHx significantly improved their surface hydrophilicity and bioactivity. Compared with pure PHBHHx, SMC enhanced the in vitro degradation and apatite-formation ability of PHBHHx in SBF. The SMC/PHBHHx-supported hMSCs show attachment and proliferation and enhance the ALP activity of hMSCs compared with PHBHHx. The adsorption and release of rhBMP-2 was controlled by varying the amount of SMC in PHBHHx. In summary, SMC/PHBHHx has excellent physicochemical, biological, and drug-release properties and has many potential applications as a 3D scaffold for bone tissue engineering.

\section{Acknowledgments}

The authors wish to express their gratitude to National Basic Research Program of China (973 Program, 2012CB933600), Major Program of National Natural Science Foundation of China (No. 31330028), National Natural Science Foundation of China (No. 31271011), National Science and Technology Support Program (2012BAI17B02), and the Program for New Century Excellent Talents in University (NCET-12-0856).

\section{Disclosure}

The authors report no conflicts of interest in this work.

\section{References}

1. Wang DX, He Y, Bi L, et al. Enhancing the bioactivity of Poly(lacticco-glycolic acid) scaffold with a nano-hydroxyapatite coating for the treatment of segmental bone defect in a rabbit model. Int J Nanomedicine. 2018:1855-1865.

2. Wang Z, Li M, Yu B, Cao L, Yang Q, Su J. Nanocalcium-deficient hydroxyapatite-poly (e-caprolactone)-polyethylene glycol-poly (e-caprolactone) composite scaffolds. Int J Nanomedicine. 2017: 3123-3131.

3. Liu X, Smith LA, Hu J, Ma PX. Biomimetic nanofibrous gelatin/ apatite composite scaffolds for bone tissue engineering. Biomaterials. 2009;30(12):2252-2258.

4. Shiozaki Y, Kitajima T, Mazaki T, et al. Enhanced in vivo osteogenesis by nanocarrier-fused bone morphogenetic protein-4. Int J Nanomedicine. 2018:1349-1360. 
5. Salgado AJ, Coutinho OP, Reis RL. Bone tissue engineering: state of the art and future trends. Macromol Biosci. 9 2004;4(8):743-765.

6. Gunatillake PA, Adhikari R. Biodegradable synthetic polymers for tissue engineering. Eur Cell Mater. 2003;5:1-16; discussion 16.

7. Su J, Cao L, Yu B, et al. Composite scaffolds of mesoporous bioactive glass and polyamide for bone repair. Int J Nanomedicine. 2017: 2547-2555.

8. Middleton JC, Tipton AJ. Synthetic biodegradable polymers as orthopedic devices. Biomaterials. 2000;21(23):2335-2346.

9. Yun HS, Kim SH, Khang D, Choi J, Kim HH, Kang M. Biomimetic component coating on 3D scaffolds using high bioactivity of mesoporous bioactive ceramics. Int J Nanomedicine. 2016:2521-2531.

10. Li H, Chang J. Preparation and characterization of bioactive and biodegradable wollastonite/poly(D,L-lactic acid) composite scaffolds. J Mater Sci Mater Med. 2004;15(10):1089-1095.

11. Heidemann W, Jeschkeit-Schubbert S, Ruffieux K, et al. pHstabilization of predegraded PDLLA by an admixture of water-soluble sodiumhydrogenphosphate - results of an in vitro- and in vivo-study. Biomaterials. 2002;23(17):3567-3574.

12. Zhao S, LiY, Li D. Synthesis of CaO-SiO2-P2O5 mesoporous bioactive glasses with high $\mathrm{P} 2 \mathrm{O} 5$ content by evaporation induced self assembly process. J Mater Sci Mater Med. 2011;22(2):201-208.

13. Lin HM, Ma JY, Li XF, Wu X, Qu FY. A co-templated approach to hierarchically mesoporous-macroporous bioactive glasses (MMBG) scaffolds for bone tissue regeneration. J Sol-Gel Sci Technol. 2012;62(2): 170-176.

14. Yan $\mathrm{X}$, Huang $\mathrm{X}, \mathrm{Yu} \mathrm{C}$, et al. The in-vitro bioactivity of mesoporous bioactive glasses. Biomaterials. 2006;27(18):3396-3403.

15. Izquierdo-Barba I, Ruiz-Gonzalez L, Doadrio JC, Gonzalez-Calbet JM, Vallet-Regi M. Tissue regeneration: A new property of mesoporous materials. Solid State Sci. 2005;7(8):983-989.

16. Wu C, Zhang Y, Zhu Y, Friis T, Xiao Y. Structure-property relationships of silk-modified mesoporous bioglass scaffolds. Biomaterials. 2010;31(13):3429-3438.

17. Trejo CG, Lozano D, Manzano M, et al. The osteoinductive properties of mesoporous silicate coated with osteostatin in a rabbit femur cavity defect model. Biomaterials. 2010;31(33):8564-8573.

18. Wu C, Ramaswamy Y, Zhu Y, et al. The effect of mesoporous bioactive glass on the physiochemical, biological and drug-release properties of poly(DL-lactide-co-glycolide) films. Biomaterials. 2009;30(12): 2199-2208.

19. Zhu M, Zhang L, He Q, Zhao J, Limin G, Shi J. Mesoporous bioactive glass-coated poly(l-lactic acid) scaffolds: a sustained antibiotic drug release system for bone repairing. J Mater Chem. 2011;21(4): 1064-1072.

20. Schmidt-Winkel P, Lukens WW, Yang PD, et al. Microemulsion templating of siliceous mesostructured cellular foams with well-defined ultralarge mesopores. Chem Mater. 2000;12(3):686-696.
21. Kokubo T, Takadama H. How useful is SBF in predicting in vivo bone bioactivity? Biomaterials. 2006;27(15):2907-2915.

22. Rezwan K, Chen QZ, Blaker JJ, Boccaccini AR. Biodegradable and bioactive porous polymer/inorganic composite scaffolds for bone tissue engineering. Biomaterials. 2006;27(18):3413-3431.

23. Huang $Y$, Jin $X$, Zhang $X$, et al. In vitro and in vivo evaluation of akermanite bioceramics for bone regeneration. Biomaterials. 2009;30(28):5041-5048.

24. de Barros Coelho M, Magalhães Pereira M. Sol-gel synthesis of bioactive glass scaffolds for tissue engineering: effect of surfactant type and concentration. J Biomed Mater Res B Appl Biomater. 2005;75(2): 451-456.

25. Lutolf MP, Hubbell JA. Synthetic biomaterials as instructive extracellular microenvironments for morphogenesis in tissue engineering. Nat Biotechnol. 2005;23(1):47-55.

26. Arcos D, Vallet-Regí M. Sol-gel silica-based biomaterials and bone tissue regeneration. Acta Biomater. 2010;6(8):2874-2888.

27. Warren SC, Messina LC, Slaughter LS, et al. Ordered mesoporous materials from metal nanoparticle-block copolymer self-assembly. Science. 2008;320(5884):1748-1752.

28. Wang H, Li Y, Zuo Y, Li J, Ma S, Cheng L. Biocompatibility and osteogenesis of biomimetic nano-hydroxyapatite/polyamide composite scaffolds for bone tissue engineering. Biomaterials. 2007;28(22): $3338-3348$.

29. Meng DC, Erol M, Boccaccini AR. Processing Technologies for 3D Nanostructured Tissue Engineering Scaffolds. Adv Eng Mater. 2010;12(9):B467-B487.

30. Wu C, Zhang Y, Zhou Y, Fan W, Xiao Y. A comparative study of mesoporous glass/silk and non-mesoporous glass/silk scaffolds: physiochemistry and in vivo osteogenesis. Acta Biomater. 2011;7(5): 2229-2236.

31. Zhu M, Zhang LX, He QJ, et al. Mesoporous bioactive glass-coated poly(L-lactic acid) scaffolds: a sustained antibiotic drug release system for bone repairing. J Mater Chem. 2011;21:1064-1072.

32. Lin HM, Lin YH, Hsu FY. Preparation and characterization of mesoporous bioactive glass/polycaprolactone nanofibrous matrix for bone tissues engineering. J Mater Sci Mater Med. 2012;23(11):2619-2630.

33. Li B, Yoshii T, Hafeman AE, Nyman JS, Wenke JC, Guelcher SA. The effects of rhBMP-2 released from biodegradable polyurethane/ microsphere composite scaffolds on new bone formation in rat femora Biomaterials. 2009;30(35):6768-6779.

34. Hosseinkhani H, Hosseinkhani M, Khademhosseini A, Kobayashi H. Bone regeneration through controlled release of bone morphogenetic protein-2 from 3-D tissue engineered nano-scaffold. $J$ Control Release. 2007;117(3):380-386

35. Müller P, Bulnheim U, Diener A, et al. Calcium phosphate surfaces promote osteogenic differentiation of mesenchymal stem cells. J Cell Mol Med. 2008;12(1):281-291.
International Journal of Nanomedicine

\section{Publish your work in this journal}

The International Journal of Nanomedicine is an international, peerreviewed journal focusing on the application of nanotechnology in diagnostics, therapeutics, and drug delivery systems throughout the biomedical field. This journal is indexed on PubMed Central, MedLine, CAS, SciSearch ${ }^{\circledR}$, Current Contents ${ }^{\circledR} /$ Clinical Medicine,

\section{Dovepress}

Journal Citation Reports/Science Edition, EMBase, Scopus and the Elsevier Bibliographic databases. The manuscript management system is completely online and includes a very quick and fair peer-review system, which is all easy to use. Visit http://www.dovepress.com/ testimonials.php to read real quotes from published authors. 